\title{
Preparation and characterization of GA/RDX nanostructured energetic composites
}

\author{
YUANFEI LAN ${ }^{1}$, XUEBAO WANG ${ }^{2}$ and YUNJUN LUO ${ }^{1, *}$ \\ ${ }^{1}$ School of Materials Science and Engineering, Beijing Institute of Technology, Beijing 100081, China \\ ${ }^{2}$ Department of Fire Protection Engineering, The Armed Police Forces Academy, Langfang 065000, China
}

MS received 9 March 2015; accepted 25 April 2016

\begin{abstract}
Graphene aerogel (GA) with nano-porous structure was assembled through the formation of physical cross-links between graphene sheets by a facile sol-gel method and supercritical $\mathrm{CO}_{2}$ drying process. Then hexahydro-1,3,5-trinitro-1,3,5-triazine (RDX) was added and trapped in the nano-porous three-dimensional networks of GA to obtain a novel GA/RDX nanostructured energetic composite. The composition, morphology and structure of the obtained GA/RDX nanostructured energetic composite were characterized by elemental analysis, scanning electron microscopy, nitrogen sorption tests and X-ray diffraction. Moreover, the thermal decomposition characteristic was investigated by thermogravimetry and differential scanning calorimetry. The results showed that GA could be a perfect aerogel matrix for the fabrication of GA/RDX nanostructured energetic composite due to its unique nano-porous structure and attributes. It was also demonstrated that RDX homogeneously disperses in the asprepared GA/RDX nanostructured energetic composite at nanometric scale. GA showed promising catalytic effects for the thermal decomposition of RDX. After incorporating with GA, the decomposition of RDX was obviously accelerated.
\end{abstract}

Keywords. Graphene aerogel; hexahydro-1,3,5-trinitro-1,3,5-triazine; nanostructured energetic composite; sol-gel.

\section{Introduction}

Two-dimensional graphene with 1 atom thickness, a single layer of graphite packed into a dense honeycomb crystal structure has attracted a great deal of attention in recent years due to its more excellent physical properties, such as large theoretical-specific surface area [1], high electrical conductivity [2], high Young's modulus [2] and thermal conductivity [3]. Graphene aerogels (GAs) with three-dimensional network structure were assembled through the formation of physical cross-links between graphene sheets using solgel chemistry. Sol-gel chemistry involves the reduction of graphene oxide to form a highly cross-linked hydrogel, which can then be freeze-dried or supercritical- $\mathrm{CO}_{2}$-dried to form a GA [4]. GAs have high surface areas, abundant nanoscale pores, high electrical conductivity and strong mechanical strengths, which make them candidates for use in energy storage, sensing applications and as supports for catalysts [4-6].

It is well known that nano-energetic materials with distinct structures and extremely small feature sizes exhibit structure- and size-dependent properties, including thermal decomposition, sensitivity and operational performance $[7,8]$.

*Author for correspondence (yjluo@bit.edu.cn)
Therefore, the controllable preparation of nano-energetic materials with different morphologies and sizes is of great importance for achieving desirable properties. Up to now, relatively few facile and simple approaches have been developed to construct the nanoparticles of energetic materials, mainly including reprecipitation [7,9], rapid expansion of supercritical solutions [10-12] and physical vapour deposition techniques [13]. Sol-gel process is a useful and feasible way to prepare the nano-structured energetic nanocomposites under mild conditions, which enables to easily control the stoichiometry and homogeneity of the composite gel that conventional methods lack [14]. For composite energetic materials using sol-gel process, explosives are loaded into the pores of the wet monolithic gel and are kept there upon drying, which helps restrain growth of the particle beyond the nanometer and submicron scale [15]. Nanostructured energetic composites with two components prepared by sol-gel methods had been numerously reported in the past. This method has proven to be successful for preparing nanostructured energetic composites with two or three components, such as aerogel thermite composites [16-18], RDX/RF [19], CL-20/GAP [20], AP/RDX/SiO [21] and HMX/AP/RF [22] nanocomposite energetic materials. As mentioned above, GAs have unique structure and attributes, which enable them to be a perfect aerogel matrix for the fabrication of nanostructured energetic composites. Adding hexahydro-1,3,5-trinitro-1,3,5-triazine (RDX) into 
the porous network of GAs can produce a novel GA/RDX nanostructured energetic composite by sol-gel method.

\section{Experimental}

\subsection{Materials}

The raw RDX (industrial products) was obtained from Baiyin Yinguang Chemical Material Factory of China. Graphite powder was provided by Beijing Chemical Reagents Company Co., Ltd. Phosphorus pentoxide was purchased from Beijing Jingqiu Chemical Industry Co., Ltd. Sulphuric acid, potassium persulphate, potassium permanganate, hydrogen peroxide solution (30\% aq.), hydrochloric acid, L-ascorbic acid and N,N-dimethylformamide (DMF) were products from Beijing Chemical Works. All of these reagents were used without further purification.

\subsection{Preparation of graphene oxide and graphene hydrogels}

The graphene oxide used in this study was prepared from natural graphite according to the modified Hummers methods [23]. In brief, graphite powder $(6 \mathrm{~g})$ was put into a solution of concentrated $\mathrm{H}_{2} \mathrm{SO}_{4}(30 \mathrm{ml}), \mathrm{K}_{2} \mathrm{~S}_{2} \mathrm{O}_{8}(5 \mathrm{~g})$ and $\mathrm{P}_{2} \mathrm{O}_{5}$ $(5 \mathrm{~g})$ for $5 \mathrm{~h}$ at $80^{\circ} \mathrm{C}$. After the temperature was cooled to room temperature, the mixture was then carefully diluted with distilled water and filtered. The product was dried in $80^{\circ}$ oven overnight. Next, pre-oxidized graphite powder was added to concentrated $\mathrm{H}_{2} \mathrm{SO}_{4}(230 \mathrm{ml})$ in an ice-bath and later $\mathrm{KMnO}_{4}(30 \mathrm{~g})$ was slowly added while stirring. The ice bath was then removed and the system was heated at $35^{\circ}$. After $2 \mathrm{~h}$, water was slowly added to the system and it was stirred for another $2 \mathrm{~h}$. Afterwards, $\mathrm{H}_{2} \mathrm{O}_{2}$ were added to reduce the residual $\mathrm{KMnO}_{4}$ till no bubble appeared. After filtering and washing, graphite oxide was obtained.

Graphene hydrogels were assembled through the formation of physical cross-links between graphene sheets using sol-gel chemistry. Sol-gel chemistry involves the reduction of graphene oxide to form a highly cross-linked hydrogel, which can then be freeze-dried or supercritical- $\mathrm{CO}_{2}$-dried to form a GA. In a typical synthesis to prepare graphene oxide directly from graphite oxide, stable graphene oxide aqueous solution was obtained by sonicating the aqueous suspension of graphite oxide for 1.5-2 h under ultrasonication conditions. The quantitative L-ascorbic acid was mixed uniformly with the above suspension for reduction of graphene oxide into graphene, and then the graphene hydrogel was obtained by heating the mixture without stirring for $16 \mathrm{~h}$. The as-prepared hydrogel was washed with a large amount of deionized water to remove residual L-ascorbic acid and its oxidized product dehydroascorbic acid.

\subsection{Preparation of GA/RDX nanostructured energetic composites}

The as-prepared clean graphene hydrogel was further replaced by DMF to remove the water within the network of the hydrogel. After that, the gel was immersed with saturated DMF solution of RDX at different temperatures (20, 45 and $65^{\circ} \mathrm{C}$ ) in a beaker for 5 days. Afterwards, the gel containing RDX was further replaced by alcohol for 3 days to replace DMF within the network of the gel. Finally, in a typical procedure, the gel was dried with supercritical $\mathrm{CO}_{2}$ to obtain GA/RDX nanostructured energetic composites for further characterization. The synthetic route for GA/RDX nanostructured energetic composites is illustrated in figure 1.

For brevity, the GA/RDX nanostructured energetic composites prepared at 20,45 and $65^{\circ} \mathrm{C}$ are labelled as R-20, R-45 and R-65, respectively.

\subsection{Measurements and characterizations}

2.4a XPS analysis: X-ray photoelectron spectroscopy (XPS) was recorded on a PHI Quanteral II spectrometer (Japan) using $\mathrm{Al} \mathrm{K}$ excitation source.

2.4b Elemental analysis: The nitrogen content of GA/RDX nanostructured energetic composites was measured on a Vario EL elemental analyzer (Elementar Analysensysteme GmbH, Germany).

2.4c XRD analysis: The X-ray diffraction (XRD) patterns of the resulting samples were recorded on a X'Pert Pro MPD (PANalytical, The Netherlands) diffractometer with monochromatic $\mathrm{Cu} \mathrm{K} \alpha$ radiation $(\lambda=1.5406 \AA)$ at $40 \mathrm{kV}$ and $40 \mathrm{~mA}$, the scanning speed was $0.01^{\circ} \mathrm{s}^{-1}$ and the step size was $0.01^{\circ}$ from $10^{\circ}$ to $60^{\circ}(2 \theta)$.

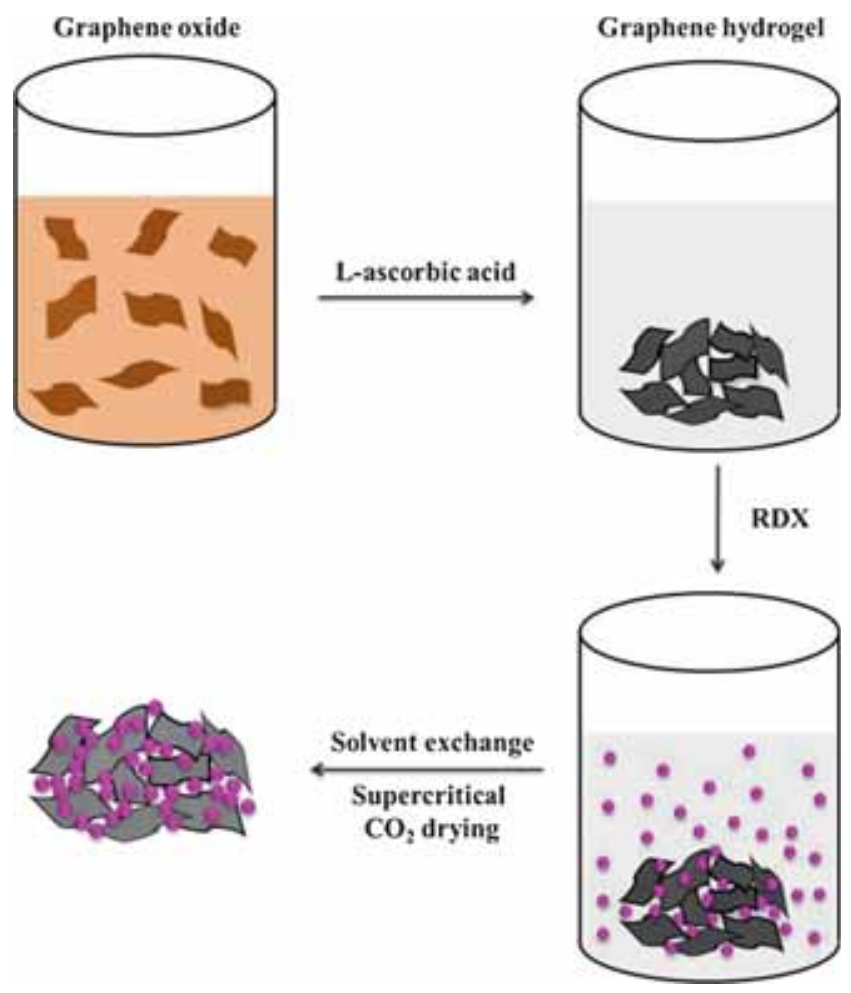

Figure 1. Schematic illustration of the synthetic route for GA/RDX nanostructured energetic composites. 
2.4d Morphological analysis: The morphology of GA and GA/RDX nanostructured energetic composites, after coating with gold, were observed with a HITACHI S-4800 field emission scanning electron microscope (SEM) at an accelerating voltage of $15 \mathrm{kV}$.

2.4e Nitrogen sorption tests: The pore structure of the samples was characterized by physisorption of nitrogen at $77 \mathrm{~K}$, which was measured in the ASAP 2020 volumetric analyzer (Micromeritics Inc., GA). Prior to measurements all samples were degassed at $100^{\circ} \mathrm{C}$ for at least $10 \mathrm{~h}$. Specific surface area was determined by the standard BET (BrunauerEmmet-Teller) method from $\mathrm{N}_{2}$ sorption. The total pore volume $\left(V_{\text {tot }}\right)$ was estimated from the amount adsorbed at a relative pressure of $\sim 0.99$.

2.4f Thermal analysis: The thermal properties of the samples were investigated using differential scanning calorimetry (TGA-DSC1-1100LF, Mettler Toledo, Switzerland). About $1 \mathrm{mg}$ of the sample was put in an alumina
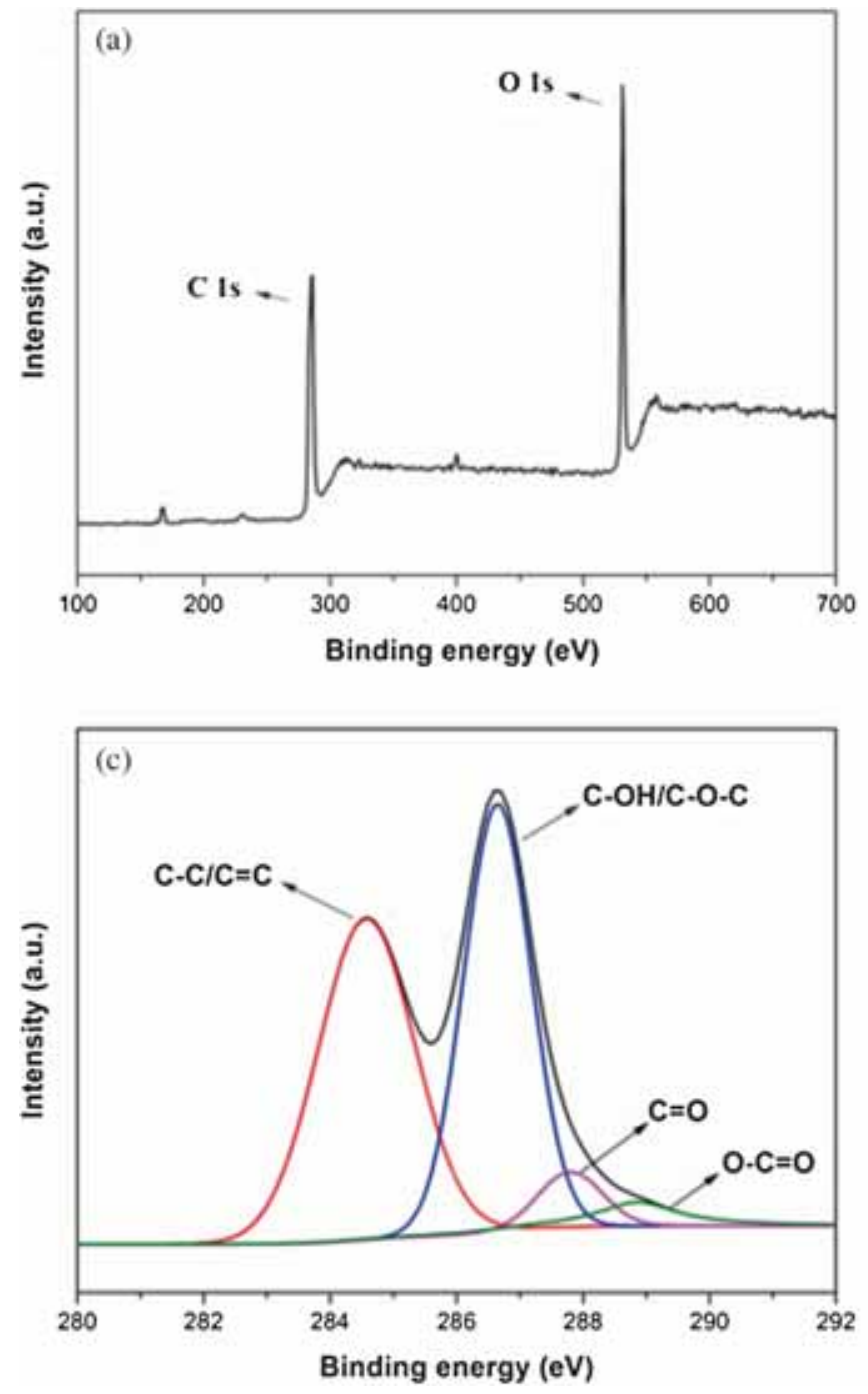

crucible and heated from 30 to $600^{\circ} \mathrm{C}$ at a heating rate of

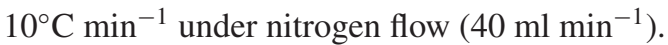

\section{Results and discussion}

\subsection{XPS characterization}

As shown in figure $2 \mathrm{a}$, the $\mathrm{C} 1 \mathrm{~s}$ and $\mathrm{O} 1 \mathrm{~s}$ peaks appear in the survey spectra of graphene oxide, indicating that some carbon double bonds are oxidized during the oxidation process. Although the survey spectra of GA exhibits same characteristic peaks of $\mathrm{C}$ and $\mathrm{O}$ elements, shown in figure $2 \mathrm{~b}$, the relative intensity of $\mathrm{C} 1 \mathrm{~s}$ peak increases while that of $\mathrm{O} 1 \mathrm{~s}$ peak decreases significantly in comparison with graphene oxide. Furthermore, four type of carbons are detected and shown in figure $2 \mathrm{c}$ and $\mathrm{d}$. The peaks centred at 284.6, 286.5, 287.8 and $289.0 \mathrm{eV}$ correspond to $\mathrm{C}-\mathrm{C} / \mathrm{C}=\mathrm{C}, \mathrm{C}-\mathrm{OH} / \mathrm{C}-\mathrm{O}-\mathrm{C}, \mathrm{C}=\mathrm{O}$ and $\mathrm{O}-\mathrm{C}=\mathrm{O}$, respectively. In case of $\mathrm{GA}$, the intensity of the peak centred at $286.5 \mathrm{eV}$ decreases significantly and the intensities of the peaks centred at 287.8 and $289.0 \mathrm{eV}$ are also
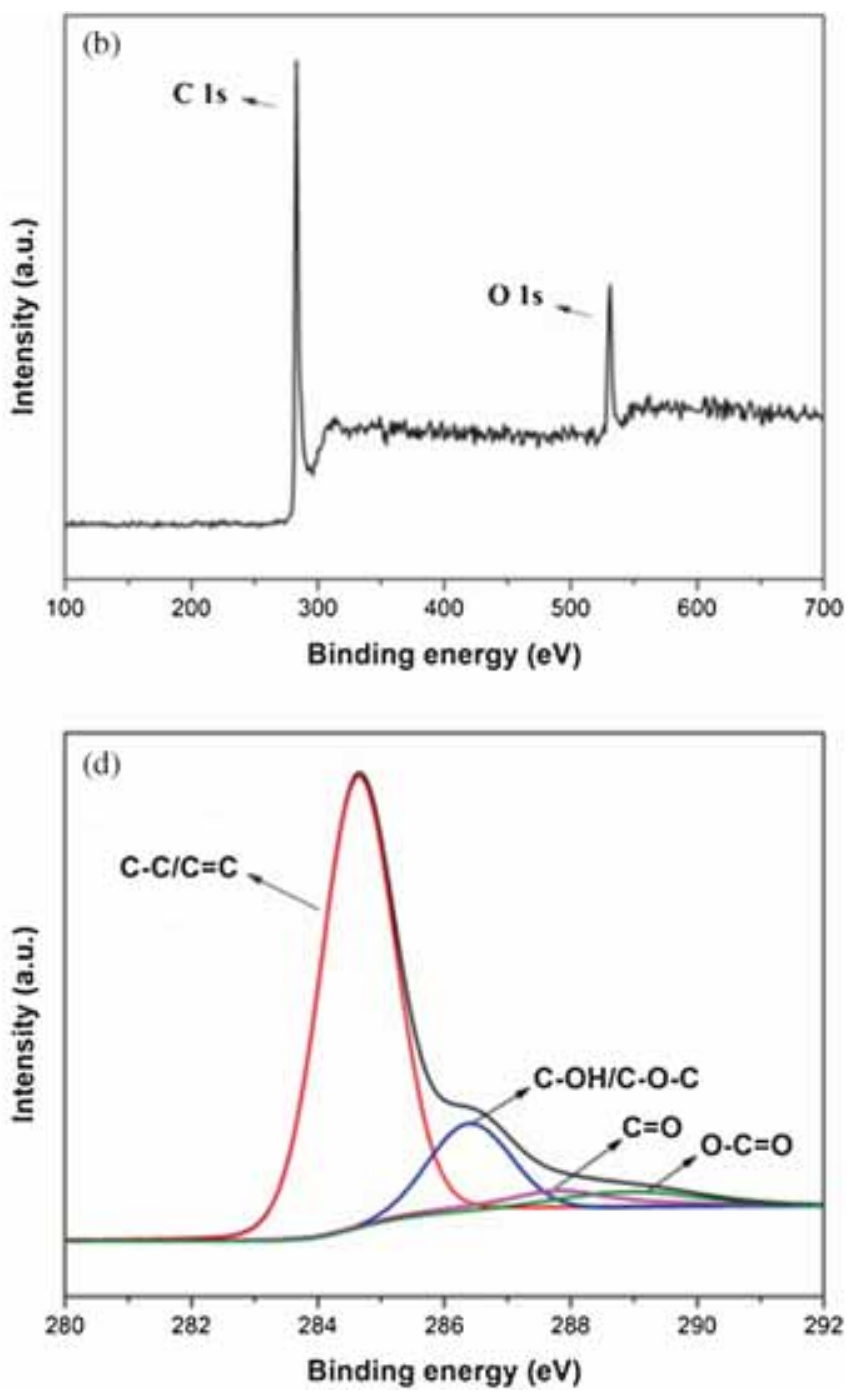

Figure 2. XPS survey spectra of (a) GO and (b) GA. C1s XPS spectra of (c) GO and (d) GA. 
much lower than those for graphene oxide. It suggests the effective elimination of the most oxygen containing groups and the restoration of the most conjugated bonds on graphene oxide after the reduction by L-ascorbic acid. As mentioned above, graphene oxide has been reduced to GA which has abundant nanoscale pores (as confirmed by SEM and nitrogen sorption tests) and could be a perfect aerogel matrix for the fabrication of nanostructured energetic composites.

\subsection{Elemental analyses}

The contents of RDX in the as-prepared GA/RDX nanostructured energetic composites were analysed by EAs for nitrogen element, which are 86.42, 94.57 and $98.07 \%$ for R-20, R-45 and R-65, respectively, and the results are also listed in table 1 .

\subsection{XRD characterization}

Figure 3 shows the XRD pattern of GA/RDX nanostructured energetic composites, and the diffraction patterns of graphene oxide, GA and RDX are provided for comparison. As shown in figure 3 , a strong and sharp peak centred at $10.7^{\circ}$ corresponds to the (001) inter-planar spacing of graphene oxide. However, in the XRD pattern of GA, the peak at the same position disappears. It suggests that graphene oxide has been reduced to graphene by L-ascorbic acid, consistent with the results given by XPS. Moreover, XRD pattern of GA shows a broad diffraction peak centred at $23.2^{\circ}$,

Table 1. Percent content of RDX in GA/RDX nanostructured energetic composites.

\begin{tabular}{llll}
\hline Sample & R-20 & R-45 & R-65 \\
Content (\%) & 86.42 & 94.57 & 98.07 \\
\hline
\end{tabular}

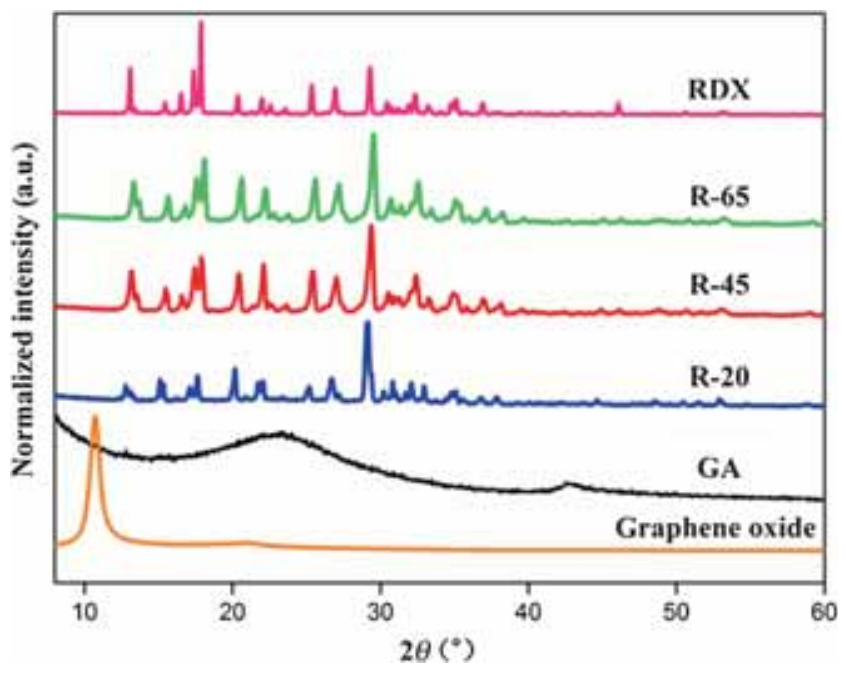

Figure 3. XRD patterns of graphene oxide, GA, RDX and GA/RDX nanostructured energetic composites prepared at different temperatures. corresponding to the (002) plane of GA [6]. For the XRD pattern of GA/RDX nanostructured energetic composites prepared at different temperatures, the same strong diffraction peaks of pure RDX appears at the same position suggesting that the incorporation process with GA has no obvious influence on the crystal form of RDX. However, the typical characteristic peak of GA at $23.2^{\circ}$ disappears in the XRD pattern of GA/RDX nanostructured energetic composites, which is probably attributed to the low mass fraction of GA in the energetic composites. The average particle diameter of RDX in the GA/RDX nanostructured energetic composites prepared at different temperatures, calculated according to the Scherrer formula, is about $71 \mathrm{~nm}$. The above results indicate that GA/RDX nanostructured energetic composites are composed of GA and RDX with average particle diameter at nanoscale, which maintains the crystalline structure of RDX and GA.

\subsection{Morphological characterization}

The morphologies of the as-synthesized materials were studied by SEM. Figure 4 shows the SEM images and photographs of GA and GA/RDX nanostructured energetic composites. As is shown in figure 4a, the morphology of GAs is quite uniform in the large scale, and GAs exhibit a threedimensional network of randomly oriented sheet-like structures with wrinkled texture, and the aerogels are rich in the hierarchical pores. This porous properties of GA were confirmed by $\mathrm{N}_{2}$ adsorption-desorption tests. From figure $4 \mathrm{~b}$, it can be seen that three-dimensional networks and porous structure exist in GA/RDX nanostructured energetic composites as observed in GA, while plenty of RDX recrystallizes and covers on the networks of the samples. As is shown in figure $4 \mathrm{c}$ and $\mathrm{d}$, both of the samples are darkish blocks and there is no apparent distinction in their appearance.

\subsection{Nitrogen sorption tests}

Nitrogen sorption tests are performed to further investigate the specific surface area and pore structure of GA and the as-obtained GA/RDX nanostructured energetic composites, which are important factors that affect catalytic properties of materials. As is shown in figure 5, $\mathrm{N}_{2}$ adsorption-desorption isotherms of the GA/RDX nanostructured energetic composites are provided and as a comparison, those of GA have also been presented. For GA/RDX nanostructured energetic composites and GA, the adsorption-desorption isotherms show a type $\mathrm{H} 3$ hysteresis loop in the middle range of $P / P_{0}$, the typical characteristic for aggregates of plate-like particles forming slit-shaped pores, which is in agreement with the results of SEM. As shown in figure 6, the pore size distribution of GA and GA/RDX nanostructured energetic composites shows that much of the pore volume lies in the nanometre size range. GA has abundant nanoscale pores, thus limiting extent growth of RDX particles. XRD analyses also confirmed that the average size of RDX was at nanoscale. 

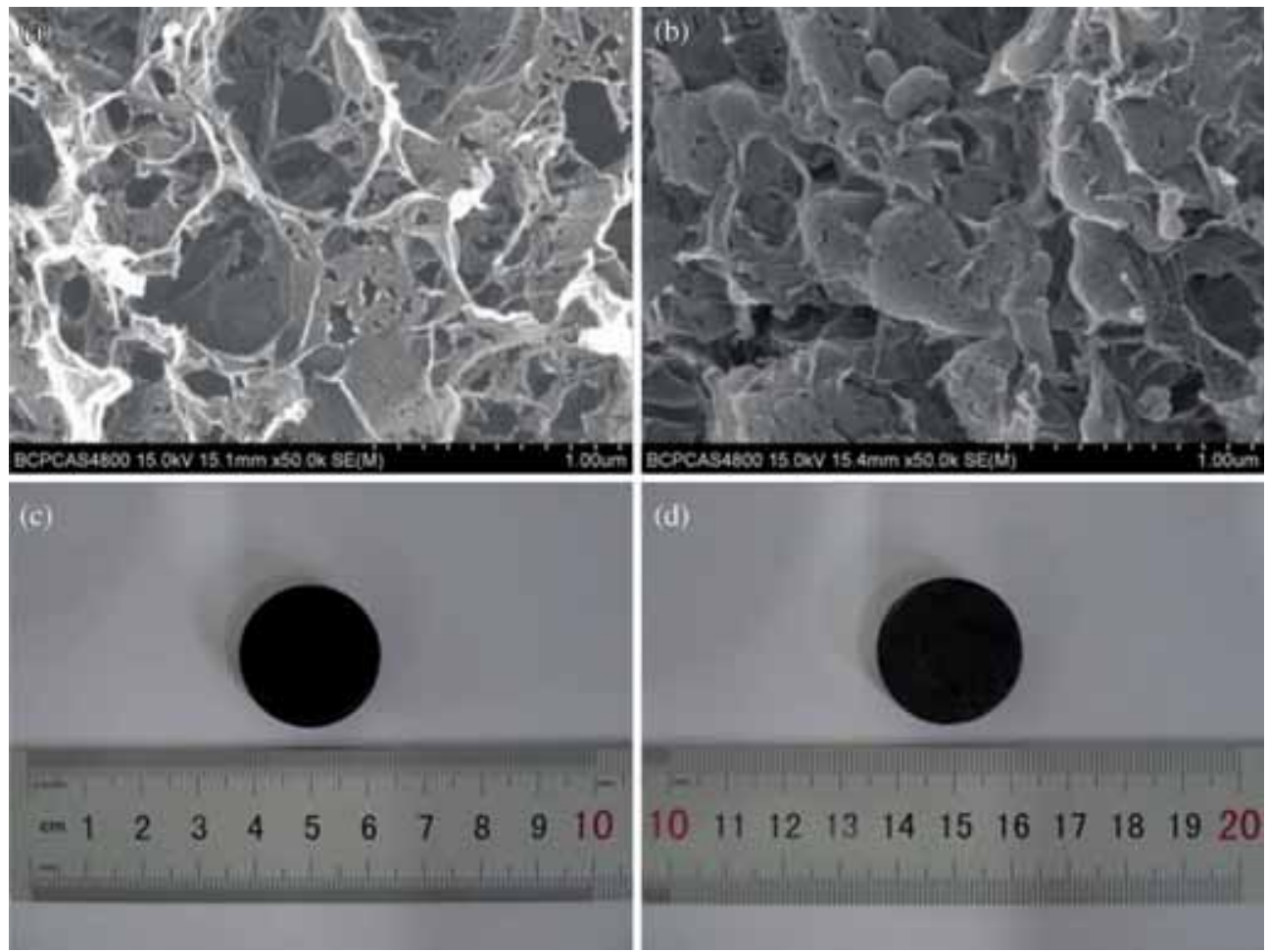

Figure 4. SEM images and photographs of (a and $\mathbf{c}$ ) GA and (b and d) GA/RDX nanostructured energetic composites.
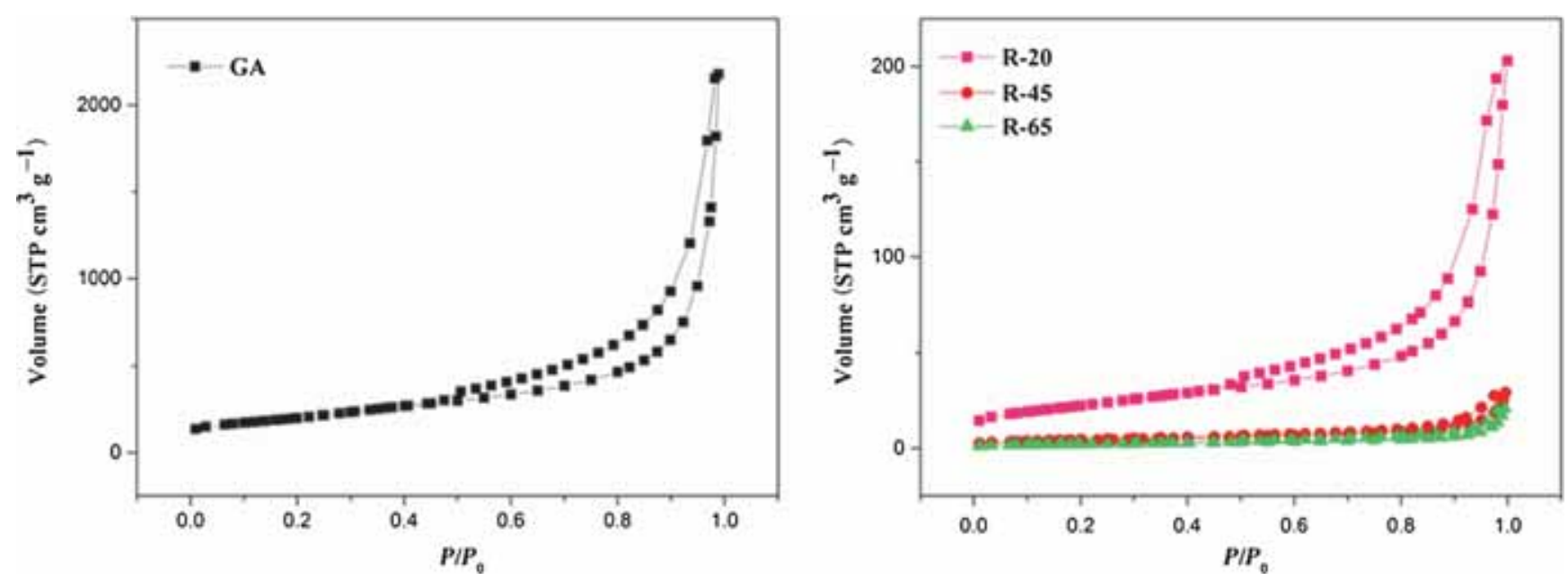

Figure 5. Nitrogen adsorption-desorption isotherms for GA and GA/RDX nanostructured energetic composites.

The results of specific surface area $\left(S_{\mathrm{BET}}\right)$ and total pore volume $\left(V_{\text {tot }}\right)$ of the samples are tabulated in table 2 . It is noticed that, after filling with RDX, R-20 shows huge decrease in the $S_{\mathrm{BET}}$ (reduced to $79.86 \mathrm{~m}^{2} \mathrm{~g}^{-1}$ ) and $V_{\text {tot }}$ (reduced to $0.31 \mathrm{~cm}^{3} \mathrm{~g}^{-1}$ ). Moreover, with the increase of amount of RDX filled, $S_{\mathrm{BET}}$ and $V_{\text {tot }}$ of GA/RDX nanostructured energetic composites gradually decrease. These decreases should be caused by the recrystallization of RDX on the graphene networks.

\subsection{Thermal analysis of the GA/RDX nanostructured energetic composites}

Thermal characterization of GA/RDX nanostructured energetic composites was performed by using thermogravimetry (TGA)-differential scanning calorimetry (DSC). An overlay of TG and DSC thermal curves of GA/RDX nanostructured energetic composites is shown along with thermal curves of pure RDX sample, and the mixture of GA with RDX sample 

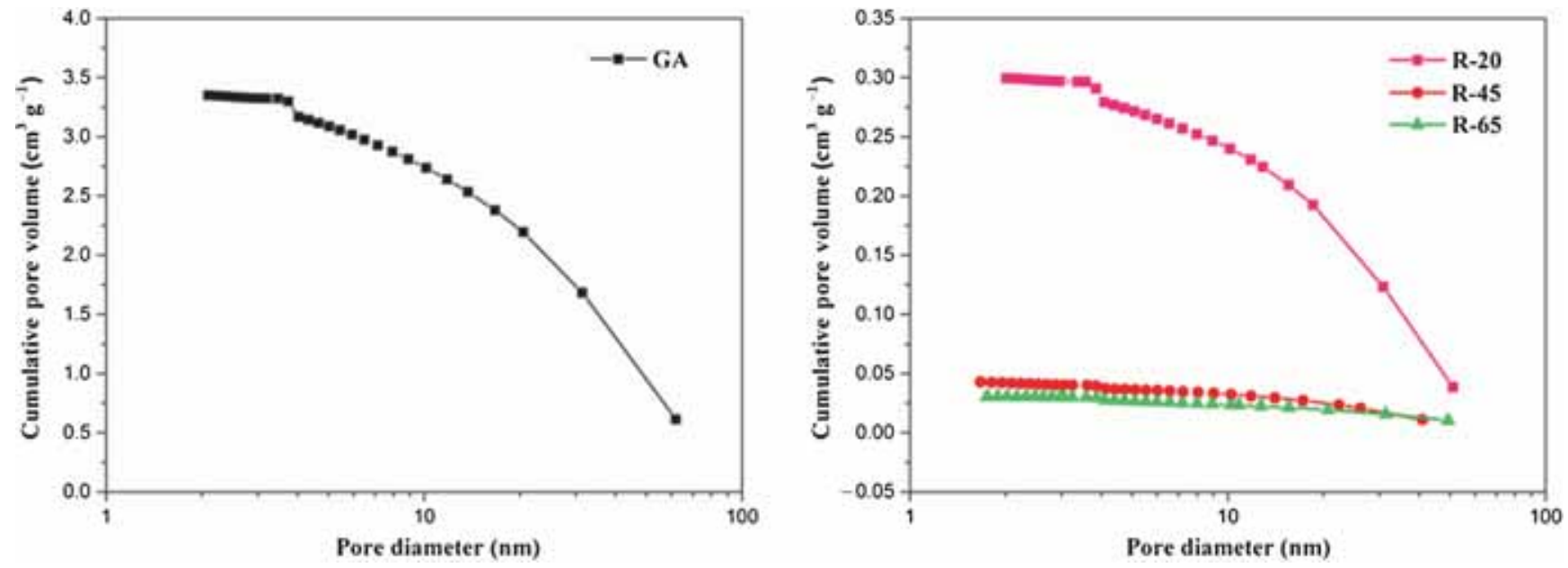

Figure 6. Desorption pore size distribution of GA and GA/RDX nanostructured energetic composites.

Table 2. Specific surface area $\left(S_{\mathrm{BET}}\right)$ and the total pore volume $\left(V_{\text {tot }}\right)$ of GA and GA/RDX nanostructured energetic composites.

\begin{tabular}{lcc}
\hline Sample & $S_{\text {BET }} / \mathrm{m}^{2} \mathrm{~g}^{-1}$ & $V_{\text {tot }} / \mathrm{cm}^{3} \mathrm{~g}^{-1}$ \\
\hline GA & 717.49 & 3.37 \\
R-20 & 79.86 & 0.31 \\
R-45 & 16.70 & 0.05 \\
R-65 & 8.44 & 0.03 \\
\hline
\end{tabular}

percentage composition was same as R-65 in figures 7 and 8, respectively. The main mass loss of pure RDX, mixture and GA/RDX nanostructured energetic composites occurred in the temperature range of $200-300^{\circ} \mathrm{C}$. In the DSC curve of RDX (figure 8), two peaks can be found. A sharp endothermic peak corresponding to melting of RDX was observed at $205.1^{\circ} \mathrm{C}$, followed by the decomposition peak at temperature of $241.7^{\circ} \mathrm{C}$. For comparison, the decomposition characterization of the mixture of GA with RDX were also investigated. It can be seen that RDX mixed with GA has effect on the decomposition temperature of RDX. The decomposition temperature of RDX mixed with GA decreased to $239.1^{\circ} \mathrm{C}$. For GA/RDX nanostructured energetic composites, their thermal decomposition temperature decreased. Moreover, with the increase of amount of GA used, the decomposition temperature gradually decreases. Compared to the decomposition temperature of pure RDX, the decomposition temperature for the sample R-20 decreases by $7.6^{\circ} \mathrm{C}$.

It can be seen from the above experimental results that GA can promote exothermic process of RDX decomposition. The decline of decomposition peak temperature means that thermal decomposition of RDX was speeded. It is beneficial to improve the burning rate. There are three ways in which GA/RDX nanostructured energetic composites improve the catalytic effect. First, GAs have an excellent heat conductive performance, which could enhance the heat conduction. Second, GAs have high specific surface area; it can absorb many reductive gas-phase products. GA can prevent the escape of

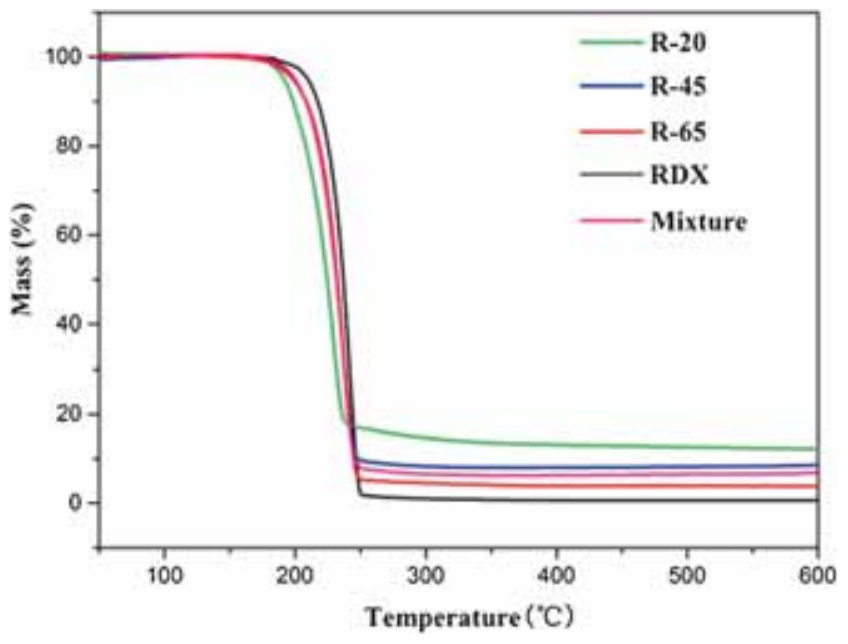

Figure 7. TG curves of pure RDX, R-20, R-45, R-65 and the mixture of GA and RDX.

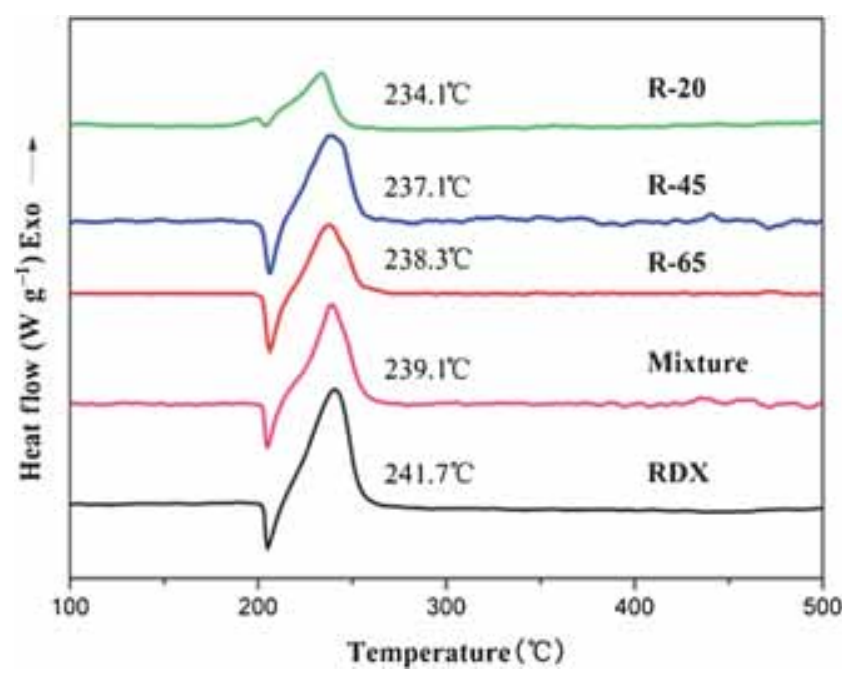

Figure 8. DSC curves of pure RDX, R-20, R-45, R-65 and the mixture of GA and RDX. 
reductive gases, ensure their full reaction in the condensed phase and play a catalytic role. Hence, catalytic effect is improved. Moreover, the nano-size of RDX in the GA/RDX nanostructured energetic composites is much smaller than pure RDX because of the GA gel skeleton. When the decomposition process takes place, the mass transfer distance of reactants is shortened.

\section{Conclusions}

In summary, a new GA/RDX nanostructured energetic composite has been successfully prepared utilizing sol-gel chemistry, whereas RDX with particle sizes in nanometre scope is added and trapped in the porous three-dimensional networks of the GA. By thermal analysis, it was proved that GA can positively catalyse the thermal decomposition process of RDX due to the unique structure and attributes. Compared to that of RDX, thermal decomposition temperature of as-prepared GA/RDX nanostructured energetic composites decreased.

\section{References}

[1] Stoller M D, Park S J, Zhu Y W, An J H and Ruoff R S 2008 Nano Lett. 83498

[2] Du X, Skachko I, Barker A and Andrei E Y 2008 Nat. Nanotechnol. 3491

[3] Balandin A A, Ghosh S, Bao W Z, Calizo I, Teweldebrhan D, Miao F and Lau C N 2008 Nano Lett. 8902

[4] Zhang X T, Sui Z Y, Xu B, Yue S F, Zhan W C and Liu B 2011 J. Mater. Chem. 216494

[5] Worsley M A, Pauzauskie P J, Olson T Y, Biener J, Satcher J H Jr and Baumann T F 2010 J. Am. Chem. Soc. 132 14067
[6] Chen W F and Yan L F 2011 Nanoscale 33132

[7] Zhang Y X, Liu D B and Lv C X 2005 Propell. Explos. Pyrot. 30438

[8] Khasainov B A, Ermolaev B S, Presles H-N and Vidal P 1997 Shock Waves 789

[9] Kaur J, Arya V P, Kaur G, Gupta Y P, Verma M M and Lata P 2010 Propell. Explos. Pyrot. 35487

[10] Pourmortazavi S M and Hajimirsadeghi S S 2005 Ind. Eng. Chem. Res. 446523

[11] Stepanov V, Elkina I B, Matsunaga T, Chernyshev A V, Chesnokov E N, Zhang X, Lavrik N L and Krasnoperov L N 2007 Int. J. Energ. Mater. Chem. Propul. 675

[12] Matsunaga T, Chernyshev A V, Chesnokov E N and Krasnoperov L N 2007 Phys. Chem. Chem. Phys. 95249

[13] Pivkina A, Ulyanova P, Frolov Y, Zavyalov S and Schoonman J 2004 Propell. Explos. Pyrot. 2939

[14] Tillotson T M., Gash A E, Simpson R L, Hrubesh L W, J H Satcher Jr and Poco J F 2001 J. Non-Cryst. Solids 285338

[15] Tillotson T M, Hrubesh L W, Simpson R L, Lee R S, Swansiger R W and Simpson L R 1998 J. Non-Cryst. Solids 225358

[16] Plantier K B, Pantoya M. L. and Gash A E 2005 Combust. Flame 140299

[17] Prentice D, Pantoya M L and Gash A E 2006 Energ. Fuel 20 2370

[18] Gao K, Li G P, Luo Y J, Wang L, Shen L H and Wang G 2014 J. Thermal. Anal. Calorim. 11843

[19] Zhang J, Yang G C and Nie F D 2010 Chinese J. Energ. Mater. 18643

[20] Li J and Brill T B 2006 Propell. Explos. Pyrot. 3161

[21] Chen R J, Luo Y J, Sun J and Li G P 2012 Propell. Explos. Pyrot. 37422

[22] Nie F D, Zhang J, Guo Q X, Qiao Z Q and Zeng G Y 2010 J. Phys. Chem. Solids $\mathbf{7 1} 109$

[23] W S Hummers Jr and Offeman R E 1958 J. Am. Chem. Soc. 801339 